\title{
L'appel au soulèvement et à la révolution par les intégristes algériens
}

Algerian Islamic fundamentalists' call for uprising and revolution

El llamado al motín y a la revolución por los integrista argelinos

\section{Latifa Ben Mansour}

\section{OpenEdition}

Journals

Édition électronique

URL : https://journals.openedition.org/mots/10483

DOI : $10.4000 /$ mots. 10483

ISSN : 1960-6001

Éditeur

ENS Éditions

Édition imprimée

Date de publication : 1 juillet 2002

Pagination : 81-93

ISBN : 2-84788-011-9

ISSN : 0243-6450

\section{Référence électronique}

Latifa Ben Mansour, "L'appel au soulèvement et à la révolution par les intégristes algériens », Mots.

Les langages du politique [En ligne], 69 | 2002, mis en ligne le 13 mai 2008, consulté le 23 avril 2022.

URL : http://journals.openedition.org/mots/10483 ; DOl : https://doi.org/10.4000/mots.10483

\section{(c) ENS Éditions}




\section{L'appel au soulèvement et à la révolution par les intégristes algériens}

Il est encore courant de lire que les autorités algériennes n'auraient jamais dû interrompre le processus des élections législatives de décembre 1992 dans leur pays, dans la mesure où le parti intégriste, le Front Islamique du Salut (FIS) légalisé en 1989, aurait pu finalement se diluer dans un fonctionnement démocratique. On lit aussi, ici et là, que les islamistes sont non pas un parti totalitaire, mais un parti qui se revendique de l'Islam. On a pourtant pu montrer que l'ensemble de la stratégie des intégristes rappelle celle des mouvements totalitaires ${ }^{1}$. Leur projet, exposé publiquement, est d'abattre l'État algérien, de traquer tout Algérien se disant laïque, démocrate ou apolitique et surtout tout musulman, pratiquant une « foi de charbonnier », mais qui refuse d'adhérer à leur parti. J'analyserai ici, sous un angle lexical et sémantico-référentiel, un certain nombre de productions discursives des dirigeants du FIS, Abassi Madani et Ali Benhadj ${ }^{2}$, durant la période où ce parti a été légalisé. J'essaierai de montrer que nous avons affaire à un discours en révolution dont les fondements ne sont pas religieux mais populistes, fascistes et antisémites. Le but de ce parti est la destruction d'un ordre établi, $A l$ Watan: (la patrie) et l'établissement d'un ordre nouveau, la Oumma (communauté des croyants), où toute différence doit être bannie et combattue, où le président, dirigeant politique élu, doit être remplacé par l'émir, le sauveur, le guide et le calife. Dès l'origine de leur mouvement, les intégristes appelaient en réalité au renversement du pouvoir en place,

\footnotetext{
- CNRS / ENS-LSH, 15, Parvis René Descartes, 69660 Lyon.

1. Voir L. Ben Mansour, 2002, Frères musulmans, frères féroces, Éditions Ramsay.

2. Je m'appuierai essentiellement sur les prêches émis par les deux leaders du Front Islamique du Salut dans les mosquées et les stades d'Alger et de sa banlieue de 1989 à 1991. Ce corpus, traduit en français par nos soins, est disponible, tout comme le programme politique du FIS, au laboratoire « Analyse de Corpus : langues, textes et apprentissages », ENS-LSH, Lyon.
} 
soit par le biais de leur 《 politique scientifique $»^{3}$ ou par celui de l'instabilité nationale (Al Fitna) en instituant la délation, la désignation du bouc-émissaire, l'appel au meurtre, et enfin la "guerre sainte ». Le remède qu'ils veulent apporter est le retour vers un «âge d'or préhistorique » de l'Islam, celui de la prédication du prophète Mohammad, du combat qu'il a mené contre les Quraysh (les habitants de la Mecque), et contre les polythéistes de Médine. Leur projet est d'établir à partir de l'Algérie un califat identique aux premiers temps "glorieux » de l'Islam, dont ils seraient bien entendu les prophètes et califes, acteurs de cette « geste », de « cette mission divine ».

Le programme politique de ce parti est instructif à plus d'un titre. Il est la façade honorable du mouvement, rédigé dans une langue de bois, particulièrement perceptible dans l'introduction. Il y est ainsi écrit : « Le sérieux est l'une des caractéristiques essentielles de l'engagement islamique actif. Pour acquérir ce sérieux il faut se conformer à la charia, à la raison, à l'intérêt et au réel. Il faut se fier à la nature humaine qui est le fruit de notre action ». Le projet développé ensuite se situe, selon ses auteurs, dans un cadre idéologique islamique qui s'articule autour de plusieurs axes, politique, économique, social, culturel et civilisationnel, militaire, extérieur. Les auteurs soulignent que leur « projet est scientifique » et proposent des critères méthodologiques pour l'élaboration "d'un plan politique scientifique de La charia». Dans le chapitre consacré à l'axe politique, il est écrit :

Pour en finir avec le despotisme, le Front adopte la choura [la consultation], pour mettre fin au monolithisme politique et au monopole sur l'économie et la société. Pour briser la dialectique perverse du népotisme-frustration, le front œuvre à codifier les responsabilités, à offrir des garanties pour l'accomplissement des devoirs dus au peuple : il encourage l'esprit de travail collectif. Meilleur antidote contre l'égoïsme, la corruption et l'individualisme ${ }^{4}$.

Il me semble possible d'affirmer que les intégristes voulaient s'emparer du pouvoir de manière légale, c'est-à-dire par la voie des urnes et aussi en infiltrant tous les rouages du dit pouvoir ou par un soulèvement généralisé et une révolution, qui est l'étape ultime de leurs aspirations à régner en Algérie pour ensuite dominer le monde.

3. On reviendra plus loin sur cette expression clé du programme politique du FIS.

4. Programme politique du FIS. 


\section{Origines et fondements de « la révolution algérienne »}

Dès les années 1980, l'Algérie connait une grave crise économique, liée à la mauvaise gestion de la rente pétrolière, aux projets gigantesques jamais achevés, à la corruption d'une grande partie de la nomenklatura, au taux de chômage qui s'abat sur les jeunes sortis des universités ou exclus du système scolaire, soit plus de vingt pour cent de la population. D'autre part, soixante pour cent de la population active a alors moins de vingt-six ans et sait que son avenir est barré. Au même moment, l'Assemblée nationale FLN adopte le Code de la Famille qui rend les femmes mineures. C'est à cette époque que surgisssent au grand jour des prédicateurs et que se constituent les premiers maquis intégristes, ceux de Bouyali en 1981. Ne pouvant s'octroyer une légitimité politique, puisqu'ils agissent dans la clandestinité et n'ont pas une assise populaire, jusqu'à la légalisation de leur parti en 1989, les intégristes vont se draper du burnous du sacré et se revendiquer comme les seuls représentants et défenseurs de l'Islam dans un pays " impie et polythéiste » (kafir wa mushrik).

A première vue, les prêches des intégristes semblent être des dénonciations des "déviations » des musulmans, c'est-à-dire des Algériens n'ayant pas adhéré à leur parti. En réalité, leur discours proclame et exige l'ordre moral, l'assainissement des mœurs sociales dissolues, le combat contre l'État impie (kafir), idolâtre (taghout), pharaon, requins (quraysh $)^{5}$. C'est surtout la destruction de l'Algérie comme nation qu'ils annoncent car, comme tous les intégristes, ils ne reconnaissent ni les frontières ni les États. C'est la oumma et le califat qui priment. De ce fait, il leur faut renverser les pouvoirs qu'ils considèrent comme impies et despotiques pour instaurer le règne de Dieu sur terre.

Alors que plus de la moitié de la population algérienne vit en dessous du seuil de pauvreté, que la corruption de la nomenklatura apparait au grand jour, et qu'il y a une quasi-inexistence des droits des citoyens, les prédicateurs se posent, du haut de la chaire des mosquées, en nouveaux apôtres de l'Islam, d'un Islam purifié de « la corruption, de la dépravation, de la dissolution des mœurs $»^{6}$, se disent les «Sauveurs de l'Islam et les rédempteurs du monde ». Ils évoquent rarement la condition misérable d'une grande partie des Algériens, le chômage, l'exode rural, la

5. Quraysh est le nom totémique de la famille du prophète contre laquelle il se révolta. Il signifie « requins » et « piastres ».

6. A. Benhadj, A. Madani, corpus des prêches intégristes. 
pénurie de logements, le manque de perspectives pour trouver un emploi et autres catastrophes quotidiennes auxquelles est confronté le peuple algérien. Leur seul objectif est la destruction de l'État, ainsi ils ne manquent pas une seule occasion de s'attaquer à «l'État policier», à «l'oligarchie militaire corrompue qui s'enrichit sans vergogne», aux « laïques suppôts de l'Occident dépravé et missionnaires de la francmaçonnerie ».

\section{Une violence portée par les réseaux lexicaux}

La virulence des propos des prêcheurs intégristes, égale à l'indigence de leurs messages, se cristallise autour d'un usage particulier de quatre lexèmes : Thawra (révolution), Fitna (discorde), Harb (guerre, qui induit al assira', le combat et al ma'rakatu, la bataille), Djihad, qui n'a dans tous les contextes qu'une seule et unique signification: "guerre sainte ».

La combinaison de ces mots, qui semblent des notions historiques et théologiques, à première vue, reflète l'idéologie et le projet de société que les intégristes algériens veulent imposer aux Algériens et au monde. Pour eux, la révolution (thawra) algérienne déclenchée en 1954 a échoué parce qu'elle n'a pas pu imposer un État Islamique. A. Madani déclare :

Le peuple algérien n'a pas accepté une autre religion que l'Islam. La renaissance politique eut lieu vers les années 1930-1940, et jusqu'au début des années 1950, mais cette renaissance politique, avec tout ce qu'elle avait de nationaliste, n'a rien pu réaliser en Algérie, ni son indépendance, ni l'instauration d'un État islamique. C'est ce qui nous a poussé à défendre la révolution (thawra) et à abandonner les partis incapables de réaliser le projet du peuple algérien, au déclenchement de la révolution (thawra) de novembre, la question de la guerre sainte (Djihad) fut débattue alors ; la question de l'émir à qui on devait allégeance, fut aussi posée. L'émir doit conduire et rassembler les gens.

Le dirigeant du FIS utilise à plusieurs reprises le lexème thawra. Ce terme n'apparait qu'à la fin de la guerre d'Algérie, dans le programme de Tripoli ${ }^{7}$, pour indiquer la transformation d'une guerre (harb) d'indé-

7. Le programme de Tripoli a été adopté en juin 1962 par le Conseil national de la Révolution algérienne. C'est ce qui a déclenché la rupture entre le gouvernement provisoire de la république algérienne (GPRA) et l'État-major de l'ALN, l'armée des frontières. C'est alors la crise de juillet entre l'armée de l'intérieur et celle de l'extérieur. L'État-major de l'extérieur avec à sa tête le colonel Boumedienne finit par l'emporter après des batailles meurtrières en aout 1962. Ce programme, d'inspiration marxiste, proche de l'Union Soviétique, est alors publié pour la première fois par le Parti Communiste français et diffusé en Algérie par le journal communiste Alger Républicain. 
pendance ${ }^{8}$ en révolution (thawra) ${ }^{9}$ s'inspirant des états socialistes. A. Madani et A. Benhadj abusent de ce terme pour mieux le dévoyer. En effet, pour les intégristes, le mot révolution s'inscrit dans le champ de l'athéisme, du matérialisme, du marxisme et du complot international. Insidieusement ils l'attribuent aux Français, donc aux Occidentaux et en particulier aux Juifs. Pour eux, si l'Algérie n'a pas entièrement acquis son indépendance, si règnent encore l'injustice, la corruption et tous les maux de la terre, c'est parce qu'il n'y a pas eu le Djihad, "guerre sainte », mais qu'il y a eu révolution (thawra) reliée aux révolutions de 1789 et de 1917, initiées, selon A. Benhadj, par les Juifs.

Il y a eu la Révolution française ; ceux qui ont été à l'origine de la Révolution française sont de confession juive, ce sont les Juifs. Ils ont dit : il faut absolument séparer le religieux du politique. La religion doit être uniquement pratiquée dans l'église, mais la vie, l'économie, la société, l'éducation, l'information, la politique intérieure et extérieure, le commerce n'ont aucun lien avec la religion ${ }^{10}$.

Les termes thawra, harb, laïcisés, appartiennent au champ du profane. Ils sont donc condamnables ${ }^{11}$, raison pour laquelle les intégristes leur préfèrent djihad. Procédant par synecdoque, ils sélectionnent dans ce terme une seule signification (" guerre sainte pour se défendre ») au détriment des autres ${ }^{12}$.

Lorsqu'on analyse les prêches, on s'aperçoit qu'à chaque étape historique correspond un terme. D'abord, thawra, la révolution, puis fitna, la discorde, ensuite sira', le combat, enfin harb, qui est inachevé s'il

8. Jusqu'en 1962, date des accords d'Évian, c'est ce mot-là (Harb Attahrir : guerre d'indépendance) qui est utilisé par la majorité des Algériens, ainsi que dans les émissions radiophoniques clandestines, comme Sawt Al'Arab (La voix des Arabes), qui émettait alors à partir du Caire.

9. Le programme de Tripoli consacre un chapitre à la Révolution populaire démocratique, dont voici les premières phrases : « La guerre (harb) de libération menée victorieusement par le peuple algérien redonne à l'Algérie sa souveraineté nationale et son indépendance. Le combat n'est pas pour autant achevé. Il est appelé au contraire à se poursuivre afin d'étendre et de consolider les conquêtes de la lutte armée par l'édification révolutionnaire (thawriyya) de l'État et de la société. Les tâches de la Révolution démocratique populaire nécessitent un examen des données objectives de la réalité ».

10. Voir Corpus des prêches intégristes, Laboratoire « Analyse de corpus : langues, textes et apprentissages », ENS-LSH, Lyon.

11. Thawra (révolution), comme sira (lutte, combat), ma'raka (bataille) ont une valeur péjorative à la différence de djihad dont la valeur est méliorative.

12. De la racine arabe /J.H.D./ le terme signifie : 1- Travailler avec assiduité, avec zèle, s'appliquer à quelque chose, faire des efforts. 2- Éprouver, tenter, tourmenter, vexer quelqu'un. 3- Surcharger une bête de somme. 4- Exténuer, épuiser, priver de forces, abattre (se dit d'une maladie). 5- Faire des efforts, lutter contre les difficultés, s'aider, s'apliquer. 6- Tourmenter quelqu'un, lui faire la guerre pour le forcer à quelque chose, d'où faire la guerre aux infidèles ou aux hérétiques ou faire la guerre sainte. 7- Appel à la vraie religion, prédication et conversion par des moyens énergiques. 
n'aboutit pas et ne s'achève pas par le djihad. Ces mots, marqués sur le plan idéologique, sont énoncés en raison de l'évènement qui les a provoqués. Cet évènement est nommé par le terme Al Fitna, dont il est intéressant de reconstruire l'histoire discursive.

De la racine arabe /F.T.N./, il renvoie à une triple valeur : 1-Éprouver, essayer, mettre à l'épreuve quelqu'un. 2-Tenter, chercher à séduire, induire en tentation. 3-Exciter à la sédition, au tumulte, à la révolte. On trouve ainsi fatin, " qui soumet à l'épreuve », d'où Satan, mais aussi la beauté (homme ou femme d'une grande beauté, qui séduit, cause des rivalités et excite le trouble dans les esprits), ainsi que fitnatu, « tentation et impiété entrainant la discorde dans la communauté des croyants ». Utilisé par les intégristes, ce terme n'est pas neutre. Il renvoie à des époques historiques datées : la mort du prophète, moment où il y eut une fitna à cause de la succession du califat, puis les suites de l'assassinat du troisième calife, Othmane Ibn Affane qui entraina la guerre (harb et non pas djihad) entre les partisans de Ali Ibn Abi Talib et Mou'awwiya Ibn Soufyane et qui causa surtout le déplacement du pouvoir de Médine à Koufa, une ville d'Irak.

La seule fois où on trouve le mot djihad pour combattre la fitna, la discorde, c'est lorsque le musulman est tenté par Satan ou par la beauté d'une femme, dans ce cas, il fait le djihad, c'est-à-dire qu'il fait un effort sur lui-même pour résister, combattre ses mauvais instincts, ses désirs, et ne pas guerroyer.

L'emploi de ces termes dans le corpus des intégristes est significatif à plus d'un titre. Il rend compte du brutal passage d'une synchronie contemporaine, l'Algérie de 1989 à 1991, à une diachronie, les années 40 à 60 de l'Islam. C'est en quelque sorte comme si toutes les révoltes, tous les combats qui se sont produits en Algérie, comme si la guerre d'Algérie qu'ils nomment harb et thawra n'avait aucune valeur à leurs yeux puisqu'il n'y a pas eu djihad dans son acception métonymique : " guerre sainte, celle qu'ils doivent nécessairement faire contre le pouvoir, pour le renverser et instaurer le gouvernement de Dieu sur terre $»$. Pour les intégristes, « l'ennemi », c'est-à-dire le pouvoir, les médias, les démocrates, les syndicats, la société civile, l'Occident, est celui qui pratique Atthawra, Assira', Al harb. Par exemple, lors de la guerre du Golfe, les États-Unis et leurs alliés ont fait la guerre (harb) au « peuple frère irakien ", tandis que celui-ci a fait le djihad ${ }^{13}$. On trouve les deux termes laïques, harb et thawra, que les intégristes ne reconnaissent pas

13. Au début de la guerre du Golfe, Saddam Husseïn disait qu'il menait une guerre (harb) à l'impérialisme américain. C'est vers les années 1995 qu'il y a eu changement dans ces discours et qu'il a utilisé le mot djihad. 
ou alors, ils les utilisent (166 occ. dans le corpus de prêches) pour ravaler leurs adversaires et le pouvoir qu'ils veulent renverser pour établir le règne de Dieu en Algérie et sur terre. Voici quelques séquences où le pouvoir est évoqué par les deux chefs intégristes :

Ce pouvoir a fait pire! / Nous demandons des comptes au pouvoir!/ Les exactions du pouvoir. / Je dirai un mot sur le pouvoir. / Au musulman, il est demandé d'obéir à Dieu et non pas aux chiens. / Le pouvoir mange, vit et se prélasse. / Le pouvoir protège l'illicite. / Un pouvoir tyrannique (taghout). / Un pouvoir militaire. / Moïse fut le seul à terrasser le maléfice du pouvoir de pharaon. / Ce pouvoir n'a plus de légitimité. / Nous n'arriverons au pouvoir qu'après avoir subi de graves pertes et sacrifices.

\section{De la guerre théologique à la guerre matérielle}

Pour abattre le pouvoir, les intégristes s'inscrivent alors dans une logique guerrière. Dans un prêche à la Casbah, A. Benhadj développe ce programme sur le plan théologique :

Leur religion n'est pas celle de musulmans. La religion des musulmans nous a été dictée par Monseigneur Abraham, l'envoyé d'Allah, le grand et le majestueux. Assemblée de musulmans, nous ne voulons aucun conflit. Nous voulons seulement ouvrir les yeux des gens sur notre religion et sur notre Islam. Nous ne brandissons ni épées, ni canons, ni chars. Nous brandissons uniquement notre religion. Auriez-vous peur de l'Islam ? Oui ils en ont peur. Oui, ils nous ont isolés. Oui, ils ont peur de la religion. Parce que la religion est une guerre contre les mœurs dissolues, contre la corruption sur terre. C'est une guerre contre le clivage social. Une classe vit, mange, jouit de la vie et dans l'autre classe, personne ne mange.

Le terme harb, "guerre réelle », utilisé ici, n'apparait pas chez les sous-lieutenants du FIS ni dans le programme. En revanche, il est proféré à cinquante-trois reprises dans le corpus des prêches des deux dirigeants. Le discours intégriste semble y opérer un glissement des valeurs sémantiques du mot harb sur le mot djihad ( 25 occ.). On observe un glissement semblable entre thawra qui signifie « révolution » et djihad. Par exemple, dans le prêche de A. Benhadj, en 1989, à la Mosquée de Bab El Oued :

Ils ne cesseront de vous combattre (harb) jusqu'à ce que vous abandonniez votre religion, s'ils le peuvent. L'Histoire, mes frères, recommence de la même manière et la lutte entre l'Islam et les ennemis de l'Islam est la même. Les moyens diffèrent ! Même si les musulmans ont tout interprété de cette religion ! Ils restent toujours à la merci de calomnies et de rumeurs. Parce qu'ils délaissent le Livre de Dieu ! Nous disons en toute vérité, nous combattons (djihad) pour le Livre de Dieu! 
Si la Charia n'est pas appliquée sur la terre d'Allah (l'Algérie), si la Charia n'est pas appliquée sur la terre des musulmans, il y aura encore et encore des guerres (harb).

Ainsi le mot djihad, qui signifie en premier lieu «lutter contre ses mauvaises habitudes afin de s'améliorer », s'imprégne peu à peu, dans ces contextes, de connotations guerrières et mortifères. Exemples encore, chez A. Benhadj :

C'est une lutte (djihad) entre la vérité et le mensonge. C'est une guerre sainte (djihad) entre la Loi d'Allah et celle des hommes. C'est un djihad entre la Loi du Ciel et celle de la terre. C'est un djihad entre la Révélation d'Allah et celle de Satan. Il est demandé au peuple de défendre (djihad) les armes à la main, sa religion et sa Charia.

On observe un glissement analogue entre thawra (dix occ.) qui signifie « révolution » et djihad.

A. Madani déclare, dans le discours de juin 1991 au stade du 5 juillet :

Il n'y a pas si longtemps, tout ce peuple s'est révolté (thawra). Il est sorti vainqueur d'une guerre (harb) effroyable où il a combattu et affronté (djihad) le colonialisme français, jusqu'à ce qu'Allah lui fasse réaliser la victoire. À partir de maintenant, nous ne permettrons à aucun individu de venir dire : Moi, je suis le pharaon de mon siècle. Jamais plus! Nous nous fondons sur ce verset qui dit: «Le seul pouvoir est celui d'Allah. Le seul royaume est celui d'Allah». Sois prêt ô peuple algérien à imposer le Livre Sacré par la force (djihad). Que dirons-nous à nos frères ? Que dirons-nous à nos enfants et à nos hommes qui ont combattu (djihad) pour le triomphe du FIS ? Que leur dirons-nous? Nous leur dirons ce que nous vous avons dit : Votre peuple dans sa majorité s'est révolté (thawra) pour élever la parole d'Allah et il est combattu (harb). S'il est agressé, il fera la guerre sainte (djihad).

Une seule fois thawra est pris en charge par les intégristes, dans les propos tenus par A. Madani (souligné ci-dessus). Implicitement, le soulèvement révolutionnaire de 1954 est le fait d'athées puisqu'ils n'ont pas préalablement débattu du djihad. Implicitement, les Algériens, tous les Algériens, sont assignés à la place des colons et du pouvoir colonisateur. Ce sont des non-musulmans et dans ce cas, la guerre sainte comme le versement de leur sang deviennent licites.

Les chefs intégristes insistent tout au long de leurs oraisons, comme dans leurs discours politiques, sur leur position de " victimes ». L'image des « colonisés » obligés de combattre le «colonialisme » s'impose à l'auditoire venu écouter leurs prêches. Ils sont victimes, en premier lieu, d'un complot international. Benhadj :

Nos ennemis de l'intérieur et de l'extérieur se sont ligués contre nous. C'est un complot interne et international contre l'Islam en Algérie, contre la force islamiste en Algérie.

En second lieu d'un complot français. Benhadj toujours : 
Et si la France encouragée par les autres partis revient cette fois-ci, ce ne sera pas par la fenêtre mais par la grande porte. Par Allah, elle reviendra par la grande porte.

En troisième et dernier lieu, ils se disent les victimes d'un système corrompu, de l'injustice qui règne, des forces de sécurité, d'une politique de l'éducation jugée impie car elle consacre plus de temps à enseigner la philosophie, les mathématiques, la musique, le français, le sport, que les règles et les principes de l'Islam. Vêtu d'une apparence religieuse et sacrée, ce discours victimaire et totalitaire émet par un tour de passe-passe un appel permanent à l'insurrection et aux massacres, à travers le terme de « Révolution islamique » (Thawra islamiyya), lequel s'oppose à celles qui sont laïques, athées, nationales (Thawra wataniyya) et populaires (Thawra sha'biyya).

\section{La « révolution islamique » des intégristes algériens}

La première constatation qui ressort des exemples précédents est que, malgré la structure discursive des propos énoncés - introduction par une récitation de quelques versets du Coran, référence permanente à Allah et à son prophète Mohammad ainsi qu'aux autres prophètes reconnus par les musulmans -, le corps des prêches intégristes est d'abord une dénonciation populiste de l'injustice représentée par l'État et ses institutions, la justice, l'Éducation Nationale, la police et l'armée, une mise en accusation de l'injustice qui serait l'expression de la dépravation des mœurs, une dénonciation des Algériens « débauchés » qui n’ont pas adhéré aux thèses intégristes, un rejet fondamental des démocrates et des processus démocratiques dont les intégristes se servent pourtant pour abattre l'État algérien. A. Benhadj l'énonce clairement :

Eh bien, ils vous trompent ! Ils vous mentent. Ils se disent démocrates. Qu'est-ce qu'ils ont ces gens-là ? Ils considèrent licite ce qu'ils veulent et illicite ce qu'ils ne veulent pas. Quelle démocratie ? Et que signifie ce mot démocratie ? Je l'ai cherché dans Lisan Al Arab d'Ibn Manzur et dans Taj Al Arus ${ }^{14}$. Je n'ai pas trouvé ce mot ni son parfum. Que signifie selon eux la démocratie ? Ils disent que la démocratie a été réalisée dans la Grèce antique. C'est le pouvoir du peuple. Ce que veut le peuple, on le lui accorde ! Il veut du vin ? Bienvenue ! L'assemblée se réunit, les députés ont des maisons, des voitures, etc. Ils viennent à l'assemblée pour traiter ce genre de questions. Quelle est votre position sur le vin? Ils discutent et remettent en question la parole d'Allah. Est-ce du

14. Lisan el Arab (La langue des Arabes) et Taj Al Arus (La couronne de la mariée) sont des encyclopédies de la langue arabe rédigées pour la première par Ibn Al Manzur et pour la seconde par Azzubaydi. 
polythéisme, oui ou non? C'est le plus grand et le plus terrible des polythéismes. Comme l'a dit Allah, c'est le plus grand des péchés. Ils discutent pendant des heures et s'il y a $51 \%$ qui déclarent que le vin est licite, alors la démocratie rend licite ce qui est illicite. Voilà la démocratie qui rend licites le vin, l'usure, la prostitution, la dépravation des mœurs, la fornication (zina) et l'avortement. Voilà la démocratie ! Les 51 \% qui déclarent que le vin est licite auront raison contre les 50 \% (sic). En Amérique, ils autorisent la liberté sexuelle, la fornication et l'homosexualité au nom de la démocratie ! Nous, nous avons As-Shoura ! ${ }^{15}$ Allah nous a appris que s'il y a des paroles qui se contredisent, l'une est pour la vérité, l'autre pour le péché, on doit imposer La Vérité même si elle est minoritaire!

Deuxième constatation, derrière ce discours en trompe-l'œil, les intégristes visent d'abord l'État algérien, en appelant à une autre révolution (thawra), celle de novembre 1954 ayant selon eux échoué, puisqu'elle n'a pas été couronnée par le djihad et l'instauration d'un nouvel émir ${ }^{16}$. Pour les intégristes, le pouvoir et l'État, militaire ou non, ne sont pas légitimes, car ils n'obéissent pas aux lois de la charia. Ils vont jusqu'à effacer la profession de foi, premier pilier de l'Islam, attestant l'existence d'Allah et de son envoyé. Ils la remplacent par l'obligation du djihad, ce qui est, en soi, une hérésie.
A. Benhadj, dirigeant en second du FIS, déclare en 1989 à la Casbah :

Ils nous dirigent depuis vingt-sept ans. Depuis vingt-sept ans, ils ont des comportements erronés. Depuis vingt-sept ans, ils ne cessent de recommencer les mêmes erreurs et d'avoir des mauvais comportements. Nous, nous mettons en pratique un discours obéissant à la Charia. Quant à la constitution et à son texte, qui est légaliste, institutionnel, obéissant aux lois de la Cité, il ne nous intéresse pas. Le FIS n'est pas un parti qui obéit au pouvoir. Il obéit à Allah... Le pouvoir est militaire depuis 1962. Et il n'a pas changé. Ce sont les militaires qui prennent les décisions [...]. Il n'y a pas de pouvoir dans une société dirigée par des polythéistes. Certains moudjahidounes, pas les vrais. Les authentiques sont avec moi. Je suis leur ami et ils sont les miens. Je parle des autres qui constituent une classe dans la société. Ils sortent et te disent : Écoute voir, un peu, c'est nous qui avons libéré ce pays et vous nous insultez ? [...] Comment cela ? Nous apporter l'indépendance ? Celui qui combat, c'est dans la voie d'Allah et pour son agrément. Tu as lutté pour moi ? Et si vous n'avez pas fait la lutte armée, le jour du jugement dernier, car le djihad est une obligation comme la prière, le jeune, la zakat et le pèlerinage, vous irez en enfer. Tu as combattu ? Et le peuple alors? Qui procurait les caches, la nourriture, les boissons ? Vous auriez pu faire quelque chose sans le peuple ? Pourquoi, vous êtes des invincibles? C'est le peuple qui est le seul moudjahid. Vos moudjahidounes, s'ils n'avaient pas eu le soutien du peuple, ils n'auraient pas résisté plus de trois jours ! Qu'auriez-vous fait sans le soutien du peuple? Qu'avez-vous fait, moudjahid? Et alors? Mais à présent, c'est le peuple entier qui se soulèvera, luttera et vous combattra. Il n'y aura pas seulement un groupe ! Le peuple entier se soulèvera et vous combattra. Il n'y aura pas de tavernes mais des mosquées !

15. As-Shoura: rassemblement d'intégristes.

16. Voir à ce propos notre article dans la revue Mots, 58, décembre 1998. 


\section{A. Madani déclare quant à lui dans une conférence de presse tenue en janvier 1991 :}

La guerre (djihad) va durer! Bien. Nous voulons la paix mais il faut aussi être prêt à la guerre (djihad). Ceci est une fidélité à novembre 1954, à la guerre sainte, à cette génération et à toutes les générations de la communauté musulmane qui fait la guerre sainte dans la voie de Dieu depuis qu'a été envoyé le prophète. L'atmosphère est enthousiaste et le peuple algérien est prêt à réaliser l'espoir de novembre 1954. Dans le communiqué du premier novembre 1954, il est écrit ceci : «Nous avons pris les armes et nous ne les rendrons que lorsque nous établirons un État algérien libre et indépendant sur les bases des principes de l'Islam. Cette année, le peuple algérien instaurera cet État algérien libre et indépendant fondé sur des principes musulmans. C'est un renouvellement de notre serment et un renouveau de l'effort et de la guerre sainte dans la voie d'Allah, pour établir la Loi d'Allah dans ce pays ».

La troisième constatation est que ce discours qui prétend revenir aux sources de l'Islam est fondamentalement antisémite. Il rappelle celui de l'extrême droite nationaliste du début du siècle et celui de Jean-Marie Le Pen. Il désigne explicitement les boucs émissaires traditionnels : les francs-maçons ${ }^{17}$, l'Occident perverti, et finalement les Juifs. C'est dans ce camp des « ennemis de l'Islam » que tout Algérien qui s'oppose aux thèses intégristes est immédiatement rejeté. Voici un exemple extrait d'un prêche de A. Benhadj :

Dans l'Islam, il n'y a pas de laïcité. La laïcité est née dans les sociétés permissives et dévoyées. Elle est née dans les sociétés occidentales. La laïcité est née après la destruction de l'église. Il y a eu la Révolution française. Ceux qui sont à l'origine de cette Révolution sont de confession juive. Ce sont des Juifs ! Ils ont dit : Il faut absolument séparer le religieux du politique. La religion doit être pratiquée uniquement à l'église. Mais la vie, l'économie, la société, l'éducation, la politique intérieure et extérieure, le commerce n'ont aucun lien avec la religion. Cette pensée est européenne et juive !

A. Benhadj va jusqu'à assimiler les médias et les autres partis algériens aux Juifs du temps du prophète Mohammad :

Après les victoires éclatantes remportées grâce au prophète, les partis se sont rendu compte que s'ils restaient isolés, ils ne pourraient pas vaincre l'Islam. Ils devaient se rassembler, pourquoi ? Pour combattre l'Islam. Ceux qui ont découvert cela, ce n'étaient pas les partis, mais les Juifs, qu'ils soient maudits à jamais ! C'est ainsi que se comportent les médias du monde! Ils disent aux démocrates : il faut vous rassembler pour combattre l'Islam. Vous avez raison, car vous êtes dans votre droit puisque vous voulez la démocratie alors qu'ils veulent l'Islam. Gloire à Allah ! L'Histoire recommence, la geste du prophète se perpétue!

17. On a à peu de chose près la même structure que celle qui a été décrite par $\mathrm{O}$. Nay dans son article publié dans la revue Mots, 43, juin 1995: «La propagande antimaçonnique sous le régime de Vichy ». Les mêmes propos sont tenus par les intégristes algériens : l'internationale maçonnique, l'hydre maçonnique, les partis maçonniques-parlementaires. 
Dans ce même prêche, A. Benhadj annonce explicitement les massacres à venir :

La hache est un devoir pour affronter les tyrans. C'est un devoir pour affronter les tyrans. C'est pour cela que les partis ne doivent pas vous effrayer. Les tractations, les négociations ou le terrorisme de la pensée planifiés par les ennemis de l'Islam de l'intérieur ou de l'extérieur, en France et en Europe ne doivent pas vous effrayer. Nous dirons à la France ce qu'ont dit nos martyrs dans les montagnes : « Nous vaincrons l'oppression et les oppresseurs ». Allah nous a dit : «Vous êtes supérieurs parce que vous êtes des croyants ».

L'analyse des mots harb, thawra, fitna et djihad permet de rendre compte du fondement idéologique des intégristes algériens. Ils appellent à la révolution, mais le mot qui la désigne est connoté, "souillé par 1789 et les Juifs ». Leur justification révolutionnaire ne peut se fonder que sur le religieux, un religieux dont ils revisitent la panoplie lexicale en figeant tout terme qu'ils utilisent dans un seul trait sémantique. Ce trait devient finalement le sabre ou la hache.

On retrouve finalement des mécanismes discursifs redoutables décrits voilà plus d'un demi-siècle par Victor Klemperer, repris ensuite par Jean-Pierre Faye ${ }^{18}$ :

Car entre les mots des langues naturelles, « étrangères » les unes par rapport aux autres, à travers la production et la circulation des mots clés, quelque chose se dessine et se lie dans le chaos idéologique des langages : l'organisation sous-jacente d'une quasi-langue, d'une langue politique. Organisation qui est moins syntaxe que prosodie. Saisir cette quasi-langue ne peut se faire par le simple inventaire des lexiques. Ce pouvoir de la langue peut « redresser » le circuit économique et mener à la guerre. Cette langue-là, cette compétence, que portent ici avec des accents divers les plus obscurs récitants, il arrivera que les plus nuls d'entre eux soient les mieux placés pour la prononcer. Le langage est le plus dangereux de tous les biens. À travers lui, ici, le danger de l'histoire peut se voir.

Le terme hache n'est plus à prendre ici comme une figure de rhétorique. Cette métaphore est à entendre à la lettre.

18. J.-P. Faye, 1972, Langages totalitaires, Hermann, p. 10. 